\title{
Carcinoma anaplastico della tiroide: nuovi orizzonti terapeutici
}

\author{
Antonio Matrone ${ }^{1} \cdot$ Rossella Elisei $^{1}$
}

Accettato: 2 giugno 2021 / Pubblicato online: 14 settembre 2021

(c) The Author(s) 2021

\section{Commento a weekendo n. 134}

Il quiz n 134 "Fate presto!, http://societaitalianadiendocrino logia.it/public//pdf/quiz134.pdf, si riferiva a un uomo di 59 anni cui era stato diagnosticato un carcinoma anaplastico della tiroide BRAFV600+ di recente comparsa e con secondarismi, ma in buone condizioni generali. Gli esami strumentali mostravano la presenza di una massa cervicale indissociabile dall'istmo tiroideo di circa $8 \mathrm{~cm}$ con infiltrazione della vena giugulare e dei corpi vertebrali cervicali (da $\mathrm{C} 4$ a C7) e con metastasi polmonari. Lo spazio respiratorio risultava sufficiente, pur in presenza di una paralisi in posizione paramediana della corda vocale destra. Al quesito "Considerate le buone condizioni generali e la motivazione del paziente, l'istotipo anaplastico e la stadiazione della malattia al momento della diagnosi, qual è in prima istanza la strategia più corretta per il trattamento del paziente?", la maggior parte dei solutori (circa il 65\%) indicava una delle tre risposte indicate come non corrette e precisamente: il $30 \%$ dei solutori ha dato la risposta n. 1, "Tiroidectomia totale con asportazione dei linfonodi del collo", poco meno di un quarto ha indicato la risposta n. 2, "Tiroidectomia totale con successivo inizio delle cure palliative", una irrilevante minoranza (3\%) la n. 3. "Terapia radiometabolica con I-131". La risposta indicata come corretta (n. 4), e cioè "Trattamento radioterapico e/o farmacologico", veniva indicata da circa il $45 \%$ dei solutori.

Il carcinoma anaplastico della tiroide (ATC) è un tumore maligno, sdifferenziato per definizione, altamente aggressivo con una prognosi generalmente infausta (sopravvivenza

\footnotetext{
$\triangle$ A. Matrone

anto.matrone@yahoo.com

1 Dipartimento di Medicina Clinica e Sperimentale, Unità Operativa di Endocrinologia 1, Università di Pisa, Pisa, Italia
}

mediana di circa 6 mesi; sopravvivenza a un anno $<20 \%$ ). L'ATC si presenta in genere come una voluminosa massa in sede cervicale anteriore a partenza dalla tiroide, la quale viene sovvertita nella sua struttura, a crescita molto rapida, che invade facilmente i vasi linfatici, ematici, le strutture del collo e presenta un alto potenziale di metastatizzazione a distanza [1].

Le recenti linee guida dell'American Thyroid Association [2] suggeriscono come l'agoaspirato resti l'esame più immediato e di semplice esecuzione per la diagnosi di ATC, ma con limitato potere diagnostico (60-65\% dei casi). Di contro, la biopsia a cielo aperto è l'esame più affidabile ma non è di facile esecuzione e inoltre, determinando una vera $\mathrm{e}$ propria ferita chirurgica, rende necessario posticipare qualsiasi altro trattamento. Negli ultimi anni, l'utilizzo di una metodica poco invasiva eseguibile in anestesia locale come il core needle biopsy (CNB), che permette una caratterizzazione istologica e molecolare del tumore ottimale, sta trovando sempre più spazio per la diagnosi di ATC.

Una volta accertata la diagnosi, la valutazione iniziale di un paziente con ATC dovrebbe prevedere esami ematici di routine, funzionalità e dell' autoimmunità tiroidea e valutazione strumentale (TC total body con mdc, 18FDG PET total body, gli esami di scelta). Inoltre, data l'elevata probabilità di compressione e infiltrazione di trachea ed esofago con relativa sintomatologia, la fibrolaringoscopia e/o l'esofagoscopia andrebbero sempre eseguite.

La valutazione strumentale, che determina la stadiazione iniziale, è particolarmente importante nell'ottica del tipo di trattamento da scegliere. Nello stadio IVa (tumore intratiroideo senza metastasi linfonodali e/o a distanza) o IVb (tumore extratiroideo con metastasi linfonodali ma senza metastasi a distanza), va valutata in ambito multidisciplinare la possibilità di ottenere una rimozione completa del tumore e, in tale caso, l'intervento chirurgico dovrebbe essere preso in 
considerazione seguito dalla radioterapia esterna (entro i 6 mesi) e dall'eventuale chemioterapia [2]. Nei casi in cui, invece il tumore risulti inoperabile per l'invasione delle strutture del collo o avesse già dato metastasi a distanza, il debulking chirurgico non è consigliato, tranne in caso di ostruzione delle vie respiratorie e/o dell'esofago, e dovrebbe essere valutata la possibilità di eseguire da subito terapie locali e/o sistemiche.

Le terapie attualmente disponibili per il trattamento del ATC non operabile comprendono:

- radioterapia esterna;

- chemioterapia: Taxani (paclitaxel o docetaxel) da soli o in combinazione con Antracicline (doxorubicina) o Platino (cisplatino o carboplatino);

- terapia target: combinazione di inibitori di BRAF (Dabrafenib) e MEK (Trametinib) per i casi BRAFV600E mutati.

Se la combinazione standard radioterapia + chemioterapia convenzionale ha prodotto risultati modesti e, in genere, transitori negli ATC avanzati, risultati molto incoraggianti sono stati ottenuti con l'utilizzo dell'associazione dabrafenib e trametinib.

La presenza della mutazione di BRAF V600E, abbastanza frequente nel ATC (circa 40\% dei casi) [3], rappresenta il razionale per l'utilizzo della terapia con dabrafenib e trametinib che ha mostrato in un trial clinico prospettico, in 23 casi di ATC, una risposta completa nel $4 \%$ e una risposta parziale nel $57 \%$ dei casi, con una sopravvivenza del $80 \%$ a un anno dalla diagnosi. FDA ha approvato l'utilizzo di questa combinazione terapeutica [4]. Inoltre, questa combinazione di farmaci si è dimostrata efficace anche a scopo neoadiuvante [5] e recenti evidenze confermano un incremento importante della sopravvivenza (94\% a 1 anno) nei pazienti che hanno poi eseguito la chirurgia dopo terapia neoadiuvante [6]. Ad oggi, tale terapia in Italia non è ancora stata approvata ma può essere richiesta, dopo un'attenta valutazione del caso, per uso compassionevole.

Ulteriori terapie a target mutazionale (inibitori di NTRK, ALK, ROS1, RET), da sole o in associazione a terapia convenzionali, così come l'utilizzo dell'immunoterapia, sono ancora in valutazione all'interno di trial clinici e la loro efficacia dovrà essere valutata in ulteriori studi. Purtroppo, al momento non ci sono farmaci approvati o in sperimentazione per i casi di ATC senza alterazioni molecolari bloccabili con farmaci specifici e, in questi casi, l'unica terapia sistemica alternativa o successiva alla terapia classica è con sorafenib o lenvatinib utilizzati a scopo compassionevole $[7,8]$.

In conclusione, l'ATC è un tumore altamente invasivo che nella maggior parte dei casi infiltra in maniera importante le strutture del collo, può dare metastasi a distanza e ha una prognosi generalmente infausta. Per questo motivo, la valutazione clinica del paziente e delle sue condizioni di salute diventa un passaggio chiave nel decidere l'approccio terapeutico che dovrà prevedere, come prima cosa, la messa in sicurezza delle vie respiratorie, se necessaria. In caso il tumore fosse operabile, un approccio multidisciplinare diventa fondamentale per impostare il trattamento chirurgico e, successivamente, quello radio e chemioterapico. Nonostante l'approccio multimodale, la mortalità resta però molto elevata. Nuovi approcci terapeutici sembrano promettenti, in particolar modo quelli a bersaglio molecolare contro la mutazione di BRAF.

Funding Note Open access funding provided by Università di Pisa within the CRUI-CARE Agreement.

Conflitto di interesse Gli autori Antonio Matrone e Rossella Elisei dichiarano di non avere conflitti di interesse.

Consenso informato Lo studio presentato in questo articolo non ha richiesto sperimentazione umana.

Studio sugli animali Gli autori di questo articolo non hanno eseguito studi sugli animali.

Nota della casa editrice Springer Nature rimane neutrale in riguardo alle rivendicazioni giurisdizionali nelle mappe pubblicate e nelle affiliazioni istituzionali.

Open Access This article is licensed under a Creative Commons Attribution 4.0 International License, which permits use, sharing, adaptation, distribution and reproduction in any medium or format, as long as you give appropriate credit to the original author(s) and the source, provide a link to the Creative Commons licence, and indicate if changes were made. The images or other third party material in this article are included in the article's Creative Commons licence, unless indicated otherwise in a credit line to the material. If material is not included in the article's Creative Commons licence and your intended use is not permitted by statutory regulation or exceeds the permitted use, you will need to obtain permission directly from the copyright holder. To view a copy of this licence, visit http://creativecommons.org/licenses/by/4.0/.

\section{Bibliografia}

1. Molinaro E, Romei C, Biagini A et al (2017) Anaplastic thyroid carcinoma: from clinicopathology to genetics and advanced therapies. Nat Rev Endocrinol 13(11):644-660

2. Bible KC, Kebebew E, Brierley J et al (2021) 2021 American Thyroid Association guidelines for management of patients with anaplastic thyroid cancer. Thyroid 31(3):337-386

3. Pozdeyev N, Gay LM, Sokol ES et al (2018) Genetic analysis of 779 advanced differentiated and anaplastic thyroid cancers. Clin Cancer Res 24(13):3059-3068

4. Subbiah V, Kreitman RJ, Wainberg ZA et al (2018) Dabrafenib and trametinib treatment in patients with locally advanced or metastatic BRAF V600-mutant anaplastic thyroid cancer. J Clin Oncol 36(1):7-13

5. Cabanillas ME, Ferrarotto R, Garden AS et al (2018) Neoadjuvant BRAF- and immune-directed therapy for anaplastic thyroid carcinoma. Thyroid 28(7):945-951 
6. Maniakas A, Dadu R, Busaidy NL et al (2020) Evaluation of overall survival in patients with anaplastic thyroid carcinoma, 2000-2019. JAMA Oncol 6(9):1397-1404

7. Savvides P, Nagaiah G, Lavertu P et al (2013) Phase II trial of sorafenib in patients with advanced anaplastic carcinoma of the thyroid. Thyroid 23(5):600-604
8. Wirth LJ, Brose MS, Sherman EJ et al (2021) Open-label, single-arm, multicenter, phase II trial of lenvatinib for the treatment of patients with anaplastic thyroid cancer. J Clin Oncol 2021:JCO2003093 